\title{
Two-Touch Type Parking Slot Marking Recognition for Target Parking Position Designation
}

\author{
Ho Gi Jung, Dong Suk Kim, Pal Joo Yoon, Jaihie Kim
}

\begin{abstract}
This paper proposes a target parking position designation method based on parking slot marking recognition for automatic parking system. To reduce the search range and memory requirement, the driver designates two seed-points which are the end-point of two line-segments separating the target parking slot from adjacent parking slots. As the method does not require marking line separating parking area from roadway, it can be applied to various parking slot marking types. The method was tested with two parking slot marking types, i.e. rectangular type and 11-shape type.
\end{abstract}

\section{INTRODUCTION}

A UTOMATIC parking system consists of three components: target position designation, path planning, and path tracking by active steering.

The methods of target position designation can be divided into four categories:

1) User interface-based method [1], [2]

2) Parking slot marking-based method [3]-[5]

3) Free space detection between parked vehicles-based methods: ultrasonic sensor-based [6], [7], short range radar (SRR)-based [8], binocular stereo-based [9], [10], light stripe projection (LSP)-based [11], and scanning laser radar-based [12], [13]

4) Infrastructure-based method [14].

For parallel parking situation ultrasonic sensor-based method is the most common target position designation method. The system collects range data passing by free parking space and then registers the range data using odometry to construct the depth map of side region of the subjective vehicle.

For perpendicular parking situation, parking slot marking-based method and free space between parked vehicles-based method are complementarily combined. However, as the operation environment of automatic parking system is assumed to be urban area, parking slot marking-based method is supposed to be essential for automatic parking system coping with perpendicular parking situation.

Manuscript received January 10, 2008.

Ho Gi Jung is with the Mando Corporation Global R\&D H.Q., 413-5 Gomea-dong, Giheung-gu, Yongin-si, Kyonggi-do 449-901, Korea (corresponding author to provide phone: +82-31-300-5253; fax: +82-31-300-5496; e-mail: hgjung@mando.com; web: http://web.yonsei.ac.kr/hgjung).

Dong Suk Kim and Pal Joo Yoon are with the Mando Corporation Global R\&D H.Q. (e-mail: \{greenhupa, pjyoon\}@mando.com).

Jaihie Kim is with the School of Electrical and Electronics Engineering, Yonsei University, Seoul 120-749, Korea (e-mail: jhkim@yonsei.ac.kr).
Systems of [3] and [4] recognized parking slot marking fully automatically. In [3], the system constantly calculated virtual target position based on the vehicle position and deflection angle just before backing up. By using the virtual target position as region of interest (ROI), the system not only enhanced the recognition performance but also reduced the processing time. Then, the system eliminated the outliers and detected straight lines using an improved random sample consensus (RANSAC) algorithm, which had a high degree of robustness and was superior in calculation cost than the Hough transformation. In [4], the system made bird's eye view image from input image captured with fisheye lens and then constructed Hough space. The system detected parking slot marking line-segments using specifically designed one-dimensional filtering in the Hough space.

In our previous work [5], we proposed a one-touch type parking slot marking recognition-based target parking position designation method. The driver designated target parking position by touching one point inside a rectangular parking slot marking on touch screen-based human machine interface (HMI). Although the method drastically reduced the search range, it had one serious drawback that it could be applied only to rectangular parking slot marking type.

This paper proposes a new parking slot marking-based method, which utilizes hints from the driver like [5]. The proposed method utilizes two hints, hereafter referred to as seed-point, which are pointed out by the driver on touch screen as shown in Fig. 1. Because the proposed method is based on two seed-points, it is referred to as two-touch type hereafter. Two-touch type has two contributions. 1) Unlike

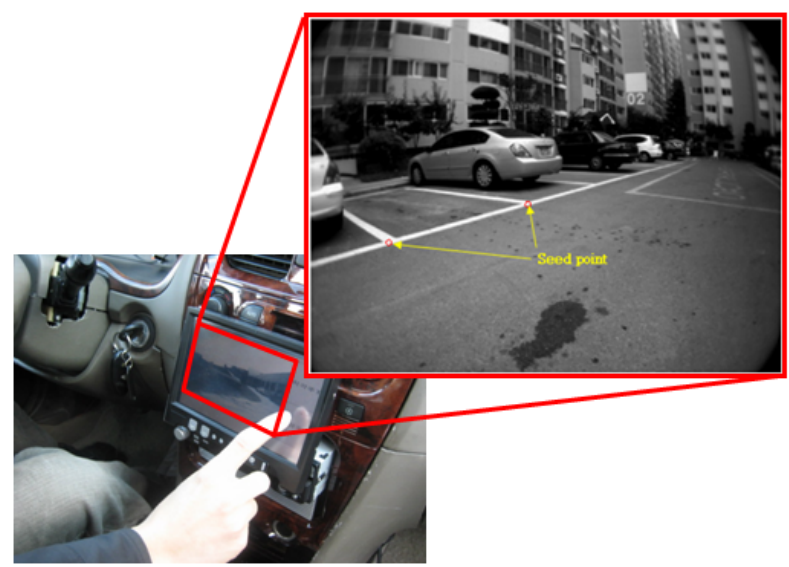

Fig. 1. Driver designates target parking position by designating two seed-points that are the end-points of parking slot marking line-segment separating neighboring parking slots. 
[5], it does not need to assume that parking slots and roadway are separated by a line. Therefore, it can be applied to various kinds of parking slot marking type. 2) As the target of recognition is pointed out directly, ROI can be established narrowly. Therefore, computational load and memory requirement for fisheye lens-related rectification and bird's eye view image construction can be reduced drastically.

\section{SYSTEM StRUCTURE}

Tow-touch type method proposed in this paper is performed following the sequence as shown in Fig. 2. 'Mode Selection' denotes the communication between the system and the driver. With the communication through touch screen-based HMI, the driver can notify the system what kind of parking operation is required. In this phase, the driver can set parallel/perpendicular selection and rectangular/11-shape selection. This paper deals with two types of parking slot marking to show the feasibility of the proposed method: rectangular type and 11-shape type.

The system captures a rearview image with rearward camera installed at the backend of the subjective vehicle, and then displays the image through touch screen-based HMI. The driver designates target parking position by pointing out two seed-points with the touch screen-based HMI.

After applying fisheye lens-related rectification and bird's eye view image transformation to the region around each seed-point, target pattern is searched. The target pattern denotes a particular pattern of parking slot marking that is supposed to exist around a seed-point. If the parking slot marking is rectangular type, then the target pattern is T-shape pattern that is the intersection between parking slot marking line-segments. If the parking slot marking is 11-shape type, then the target pattern is $\Pi$-shape pattern that is the ending of

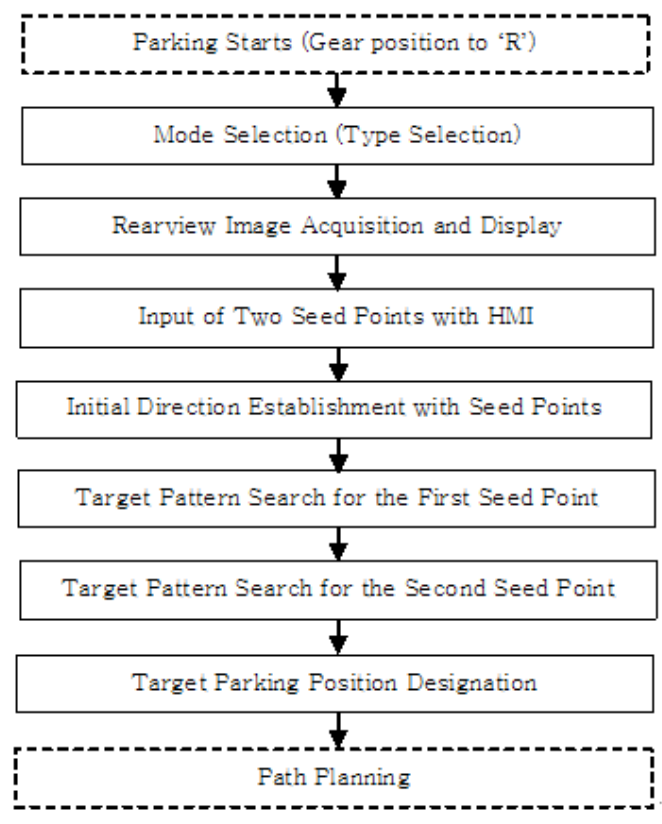

Fig. 2. The flow chart of two-touch type method. line-segment contour. In other words, target pattern detection is implemented by template matching of skeleton and contour of parking slot marking. If there is a target pattern certainly, target pattern searching is equal to finding the placement of target pattern that minimizes matching error.

Once two target patterns are detected, the coordinates of the entrance of the target parking position is fixed. Therefore, the coordinates of target parking position can be established. Consequently, based on the coordinates of target parking position, path can be planned.

\section{INITIAL DIRECTION ESTABLISHMENT}

Two seed-points, that the driver points out to designate the end-points of separating marking line-segments, are expected to be similar to the entrance of target parking position. Therefore, the longitudinal direction of target parking position can be initialized by the perpendicular direction of the line-segment connecting the seed-points. Fig. 3 shows an example of line-segment connecting two seed-points and initialized longitudinal direction of target parking position.

\section{TARgEt PATtern DETECTION}

\section{A. Target Pattern for Each Marking Type}

Once the longitudinal direction of target parking position is initialized, target pattern is searched around each seed-point. By applying fisheye lens-related rectification and bird's eye view transformation to a certain region around each seed-point, rectified image is constructed [5]. The seed-points are assumed to be located on the ground surface. Therefore, with the homography between camera installed at the backend of the subjective vehicle and the ground surface, bird's eye view image transformation can be performed. As only small region around seed-point, that is $1 \mathrm{~m} \times 1 \mathrm{~m}$, is transformed into bird's eye view image and no interpolation is used, memory consumption and computational load are restricted within a small amount.

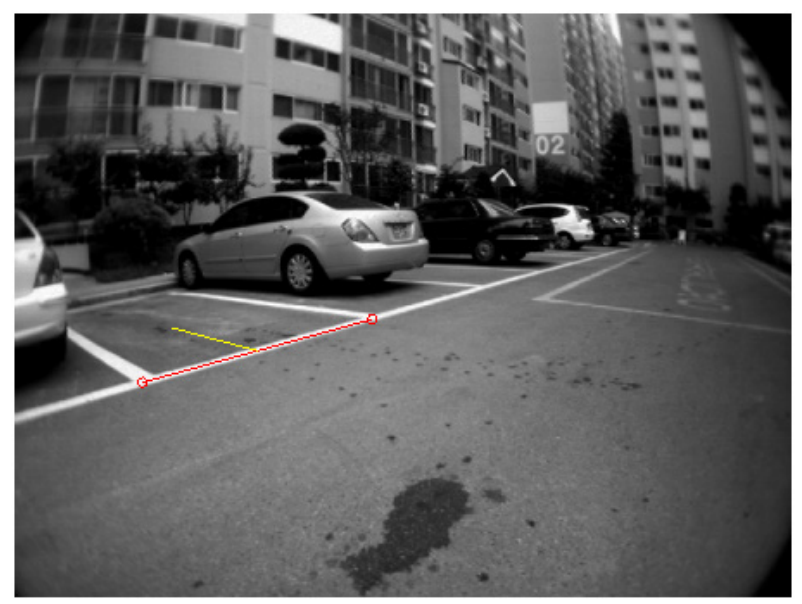

Fig. 3. The longitudinal direction of target parking position can be initialized with two seed points. 


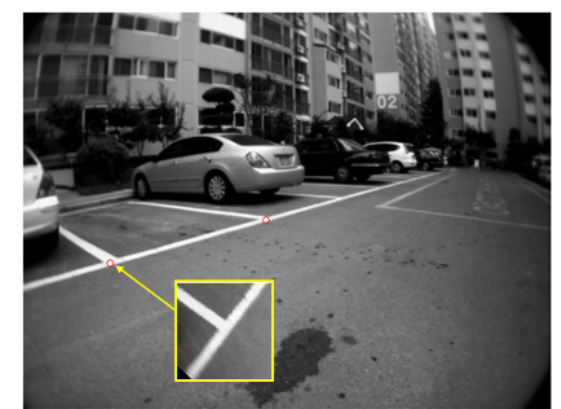

(a) Rectified image in the case of rectangular type

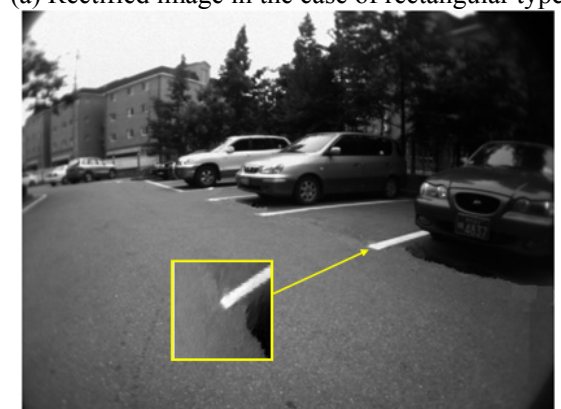

(b) Rectified image in the case of 11-shape type

Fig. 4. Rectified image for two type parking slot markings.

Fig. 4 shows the rectified images around the end-point of parking slot marking line-segment of two types of pattern. Fig. 4(a) gives an example of T-shape example. As a line separating parking area from roadway and a line-segment separating parking slots are met perpendicularly, the region around the cross-point can be modeled as a T-shape pattern. Fig. 4(b) shows the rectified image around the end-point of parking slot marking line-segment of 11-shape type, which does not have the line separating parking slots from roadway. In this case, if the central line of marking line is used alone, the position of target pattern in longitudinal direction can not be determined certainly. Therefore, in the case of 11-shape type parking slot marking, П-shape of the contour of the line-segment is selected as a target pattern.

\section{B. Distance Transform-Based Target Pattern Detection}

If it is assumed that there is one target pattern in a rectified image, then target pattern detection is equal to a problem finding the optimal coordinates and orientation of target pattern. Target pattern detection constructs the intensity histogram of rectified image, and then finds clusters in the histogram. If the number of the clusters is fixed to a sufficiently large value, e.g. four in our case, then pixel values are supposed to be over-segmented and the cluster with largest intensity value should correspond to the region of marking. Although this marking segmentation is robust to external disturbance such as asphalt texture pattern, it has one drawback that the contour of marking region contains a lot of noise. Therefore, as target pattern detection can not be implemented by syntactic approaches, proposed method uses genetic algorithm (GA)-based optimization, which minimizes errors between the distance transform of the rectified image

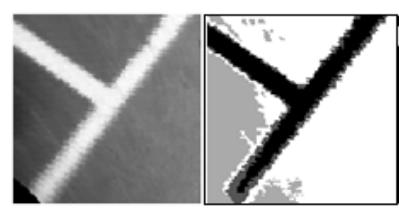

(a)

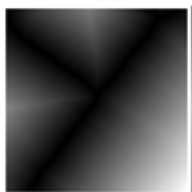

(e) (b)

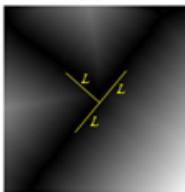

(f)

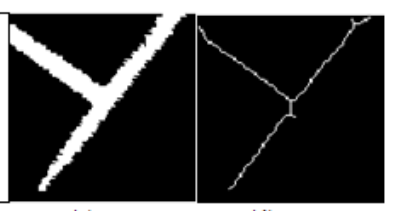

(c)

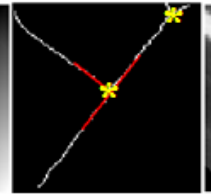

(g) (d)

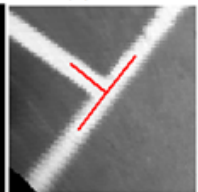

(h)
Fig. 5. T-shape target pattern detection.

and target pattern template.

1) T-shape Target Pattern for Rectangular Type

In general, as parking slot marking is drawn with light color on dark colored-ground surface, rectified image can be segmented into two kinds of region. However, considering the effect of shadow beneath vehicles and asphalt texture pattern, intensity histogram is over-clustered into four clusters and the brightest cluster is regarded as pixels belonging to parking slot marking. Although over-clustering causes noisy contour of parking slot marking, it can guarantee that the extracted pixels surely belong to parking slot marking. In the case of T-shape target pattern, binary morphological operation extracts skeleton from rectified image. When Fig. 5(a) is a rectified image, (b) shows the segmentation result and (c) shows the extracted marking region. Fig. 5(d) shows the extracted skeleton of (c).

After the skeleton is extracted, its distance transform is constructed as shown in Fig. 5(e). In the case of rectangular type, target pattern template consists of three line-segments with length $L$ as shown in Fig. 5(f). The error of a specific hypothesis, that is central coordinates and orientation, is defined as the summation of distance values that are sampled along the template line-segment by a specified interval when a target pattern template is overlapped onto the distance map according to the hypothesis.

Target pattern detection is an optimization problem minimizing the error of the placement of target pattern template, $(x, y, \theta) .(x, y)$ denotes the central coordinates of target pattern template and $\theta$ denotes the orientation of target pattern template. The proposed method solves this problem by GA. An individual consists of three genes corresponding to the placement parameters of target pattern template and the fitness function is defined as the error of the placement with respect to skeleton's distance transform. To enhance the efficiency of GA, $\theta$ is restricted to a range similar to the initial longitudinal direction of target parking position and the central coordinates of target pattern template is initialized with the cross-points of the skeleton. In Fig. 5(f), cross-points of the skeleton are depicted by '*' marking. GA is performed with population size 200 and maximum generation 100. Fig. $5(\mathrm{~g})$ and $(\mathrm{h})$ show the detected T-shape target pattern 


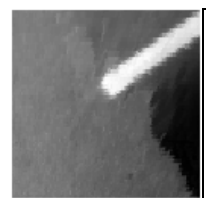

(a)

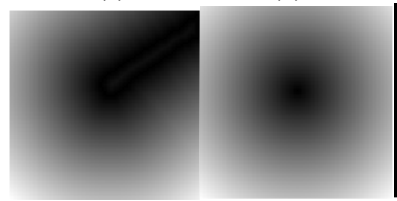

(e)

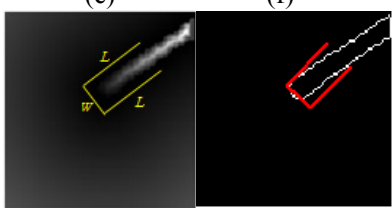

(i)

(j)

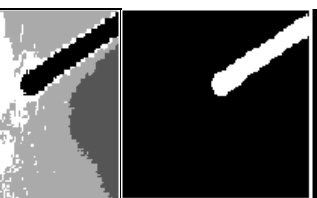

(c)

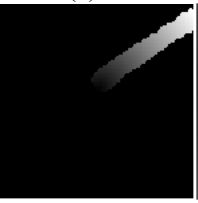

(g)

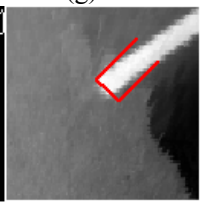

(k)
Fig. 6. П-shape target pattern detection.

respectively on the distance map and the rectified image.

2) П-shape Target Pattern for 11-shape Type

Like the T-shape target pattern detection, four clusters are detected in the intensity histogram of the rectified image as shown in Fig. 6(b) and then pixels belonging to the brightest cluster are regarded as parking slot marking as shown in Fig. 6 (c). In the case of П-shape target pattern detection, the contour of the detected parking slot marking is extracted by morphological operation as shown in Fig. 6(d). In this case, target pattern is located at the ending of the contour.

In the case of П-shape target pattern, detecting the patterns has a difficulty since the width of parking slot marking is variable. Especially, the position in the longitudinal direction is hard to be determined. To complement this difficulty, modified distance map is introduced, which considers two aspects of the line segment: contour and end-point. The modified distance map is defined as the product of contour's distance map and end-point's distance map spatially limited within detected parking slot marking. Fig. 6(e) is the distance map of the contour and (f) is the distance map with respect to the end-point of parking slot marking. Fig. 6(g) is the spatially limited distance map with respect to the end-point and (h) is the modified distance map. It can be noteworthy that the end-point of marking region has smaller value than the remaining parking slot marking in the modified distance map.

As the width of parking slot marking is not fixed, target pattern consists of three line-segments: two parallel line-segments with length $L$ and one line-segment with length $w$ connecting the two parallel line-segment's end-points perpendicularly. Then, a hypothesis consists of central coordinates, orientation and width. The error corresponding to the hypothesis is the summation of modified distance values when a target pattern template is overlapped on the modified distance map according to the hypothesis as shown

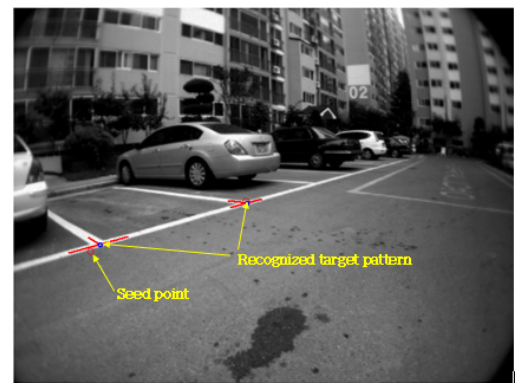

(a)

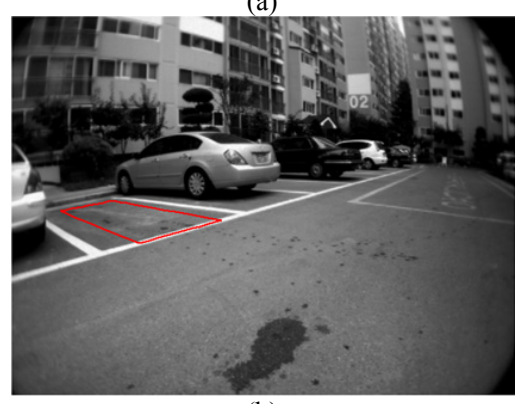

(b)

Fig. 7. Target pattern detection result and target parking position establishment result.

in Fig. 6(i). Similar to the T-shape target pattern, $\Pi$-shape target pattern is detected by GA with the same configuration. Fig. 6(j) and (k) show the detection result. Although the orientation of target pattern is wrong slightly, this can be compensated during target parking position establishment phase.

\section{TARget PARKIng Position Establishment}

If target patterns on two separating line-segments are recognized, target parking position can be established. Fig. 7(a) shows two seed-points inputted by the driver with touch screen and detection result of target patterns. Since the size of the touch screen is small and the driver's posture is limited, it is impossible to designate seed-points exactly on the target pattern. However, as the proposed method searches target patterns in the region around the seed-points, target patterns can be designated in spite of noisy driver's input. In Fig. 7(a), it is noteworthy that target patterns are exactly recognized even though the driver's seed-points are distant from the target. Therefore, the proposed method can help the driver designate target pattern without excessive stress. This is the main difference of the proposed method from the fully manual designation methods.

The target parking position can be established such that one longitudinal side of rectangular target position is on the line connecting the central points of two recognized target patterns and the center of target position is equally distant from the two target patterns. The rectangle of target parking position has the same length and width as the subjective vehicle. Even when the detected orientation of each target pattern contains some error, target parking position 

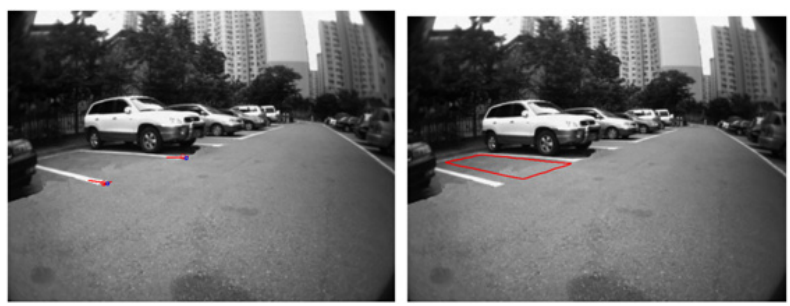

Fig. 8. Established target parking position in the case of 11-shape type.
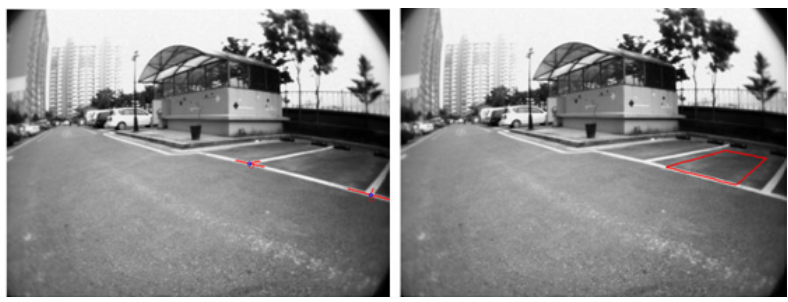

Fig. 9. Established target parking position in the case of rectangular type.

establishment method using two recognized target pattern, located at two end of entrance of parking slot, makes the position more accurate and robust. Fig. 7(b) shows the consequently established target parking position.

\section{EXPERIMENTAL RESULTS}

This paper applied the proposed method to two types of parking slot marking, e.g. rectangular type and 11-shape type. Experiments under various situations showed that the proposed method can successfully establish target parking position in practical usage.

Fig. 8 shows the recognized $\Pi$-shape target patterns and established target parking position in the case of 11-shape type. Fig. 9 shows the recognized T-shape target patterns and established target parking position in the case of rectangular type.

Especially, Fig. 10 shows the case when there is another marking line in front of target parking position. This kind of situation can not be solved by our previous one-touch type target parking position designation method of [5]. It is because two-touch type method uses only local image around the seed-point designated by the driver. Similarly, the proposed method works even when dark shadow is cast on the target pattern as shown in Fig. 11. It also shows the effect of over clustering during parking slot marking segmentation.

Fig. 12 shows the case when the target position is far from the subjective vehicle. In such a case, target pattern is distorted severely in the bird's eye view image generally. This problem disturbs the global image processing-based method of [4] because blurred and distorted parking slot markings can not be extracted. Because marking line separating parking slot from road way, named as guideline, is the key to the one-touch type method of [5], failure of guideline detection caused by blurred line image is the major factor limiting the operation range of [5].

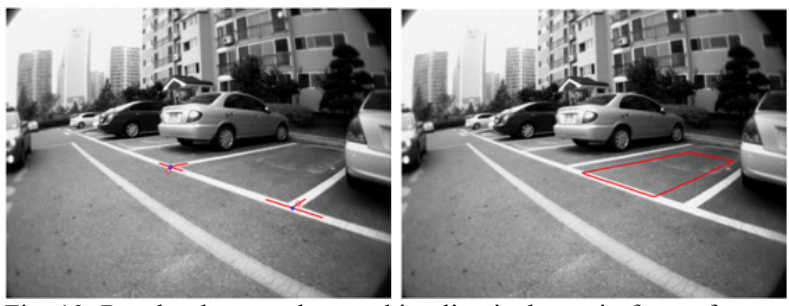

Fig. 10. Result when another marking line is drawn in front of target parking position.

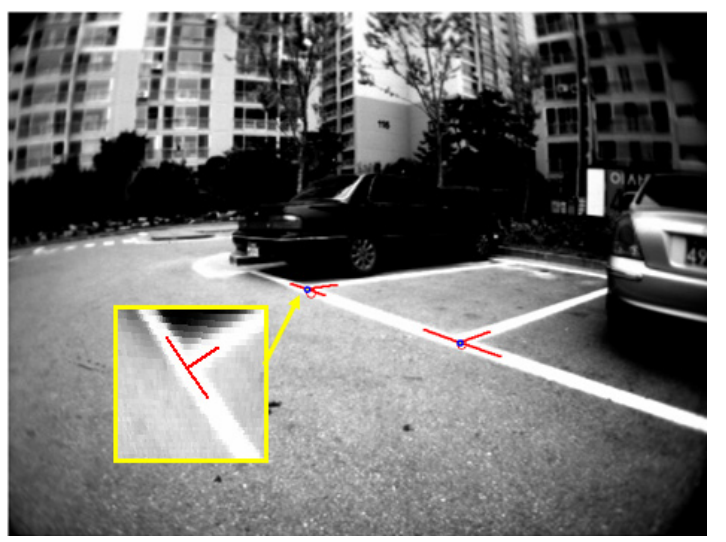

Fig. 11. Result when dark shadow is cast on the near of target pattern.

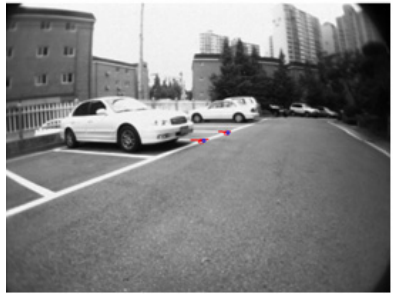

(a) Recognized target patterns
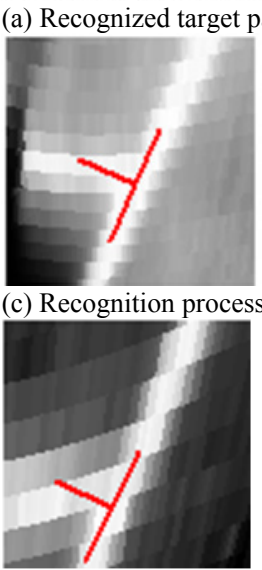

(d) Recognition process of the second target pattern

Fig. 12. Result when the target position is far.

As shown in Fig. 12 (c) and (d), target patterns are severely distorted and blurred. Even in this case, the proposed method finds marking line by over clustered segmentation and extracts parking marking region successfully. As the region of marking line is distorted, the skeleton corresponding to the region is supposed to contain severe noise as shown in Fig. 12 (c) and (d). However, as the proposed method finds the 
optimal placement of target pattern template using GA-based optimization, it can detect target pattern successfully. Furthermore, as shown in Fig. 12 (c) and (d), robustness and computation efficiency are increased by using cross-points of skeleton as the candidate of target pattern center.

\section{CONCLUSION}

This paper proposes a target position designation method for automatic parking system using rearview camera to observe the parking area and touch screen to interface with driver. In detail, with this method, the driver can establish the target parking position by designating the end-point of two marking lines between target parking slot and adjacent slots. Recognition of the end-point of marking line is the same as the recognition of the target pattern according to parking slot marking type. Therefore, if proper target pattern can be defined for each parking slot marking type, the proposed method can be applied to various parking slot marking types. In this paper, we proved the feasibility of the proposed method by applying it to two marking types, that is rectangular type and 11-shape type.

For target pattern recognition, the proposed method constructs distance map with respect to the skeleton or contour of marking line region and then find the placement of target pattern template by searching the placement parameters minimizing distance map-based error. As the error is defined using distance map, the proposed method can be applied to even when the parking slot markings are corrupted or disconnected.

Future work is developing a method that automatically recognizes the type of parking slot marking without the driver's input maneuver, i.e. parking slot marking type recognition method.

\section{REFERENCES}

[1] Masayuki Furutani, "Obstacle Detection Systems for Vehicle Safety", SAE Paper No.: 2004-21-0057.

[2] Ho Gi Jung, Chi Gun Choi, Pal Joo Yoon, and Jaihie Kim, "Novel User Interface for Semi-automatic Parking Assistance System", $31^{\text {st }}$ FISITA World Automotive Congress, Oct. 22-27, 2006.

[3] Yu Tanaka, Mitsuyoshi Saiki, Masaya Katoh, and Tomohiko Endo, "Development of Image Recognition for a Parking Assist System", $13^{\text {th }}$ World Congress on Intelligent Transportation Systems and Services, Oct. 8-12, 2006

[4] Ho Gi Jung, Dong Suk Kim, Pal Joo Yoon, and Jaihie Kim, "Pakring Slot markings Recognition for Automatic Parking Assist System", IEEE Intelligent Vehicles Symposium 2006, Jun. 13-15, 2006, pp. 106-113.

[5] Ho Gi Jung, Dong Suk Kim, Pal Joo Yoon, and Jaihie Kim, "Structure Analysis Based Parking Slot Marking Recognition for Semi-automatic Parking System", Lecture Note in Computer Science Vol. 4109, Aug. 2006, pp. 384-393.

[6] J. Pohl, M. Sethsson, P. Degerman, and J. Larsson, "A Semi-automated Parallel Parking System for Passenger Car", Proceedings of Institution of Mechanical Engineers, Part D: Journal of Automobile Engineering Vol. 220, Issue 1, Jan. 2006, pp. 53-65.

[7] Hisashi Satonaka, Masato Okuda, Syoichi Hyasaka, Tomohiko Endo, Yu Tanaka, and Toru Yoshida, "Development of Parking Space Detection Using an Ultrasonic Sensor", $13^{\text {th }}$ World Congress on Intelligent Transportation Systems and Services, Oct. 8-12, 2006.
[8] Stefan Görner and Hermann Rohling, "Parking Lot Detection with $24 \mathrm{GHz}$ Radar Sensor", $3^{\text {rd }}$ International Workshop on Intelligent Transportation, Mar. 14-16, 2006.

[9] Nico Kaempchen, Uwe Franke, and Rainer Ott, "Stereo Vision Based Pose Estimation of Parking Lots Using 3D Vehicle Models", 2002 IEEE Intelligent Vehicle Symposium, Vol. 2, Jun. 17-21, 2002, pp. 459-464.

[10] Ho Gi Jung, Dong Suk Kim, Pal Joo Yoon, and Jaihie Kim, "3D Vision System for the Recognition of Free Parking Site Location", International Journal of Automotive Technology, Vol. 7, No. 3, May 2006, pp. 361-367.

[11] Ho Gi Jung, Dong Suk Kim, Pal Joo Yoon, and Jaihie Kim, "Light Stripe Projection based Parking Space Detection for Intelligent Parking Assist System", Proceedings of the 2007 IEEE Intelligent Vehicle Symposium, Jun. 13-15, 2007.

[12] Alexander Schanz, "Fahrerassistenz sum automatischen parken", http://www.gyrosmafia.de/cms/front content.php?idcat $=74 \&$ idart $=38$ 1, Jul. 23, 2007.

[13] Ho Gi Jung, Young Ha Cho, Pal Joo Yoon, and Jaihie Kim, "Integrated Side/Rear Safety System", $11^{\text {th }}$ European Automotive Congress, May 30-Jun. 1, 2007.

[14] Massaki Wada, Xuchu Mao, Hideki Hashimoto, Mami Mizutani, and Masaki Saito, "iCAN: Pursuing Technology for Near-Future ITS", IEEE Intelligent Systems, Vol. 19, Issue 1, Jan.-Feb. 2004, pp. 18-23. 